\title{
Oceanic Restratification Forced by Surface Frontogenesis
}

\author{
Guillaume Lapeyre, Patrice Klein, and Bach Lien Hua \\ Laboratoire de Physique des Océans, IFREMER, Plouzané, France
}

(Manuscript received 3 December 2004, in final form 20 December 2005)

\begin{abstract}
Potential vorticity (PV) conservation implies a strong constraint on the time evolution of the mean density at a given depth. The authors show that, on an $f$ plane and in the absence of sources and sinks of PV, it only depends on two terms, namely, the time evolution of the product between density anomaly and relative vorticity and the vertical PV flux. This primitive equation result, which applies at any depth, suggests that the ageostrophic dynamics induced by baroclinic eddies strongly affect the mean oceanic stratification profile. This result is illustrated for two simple initial-value simulations of a baroclinic, balanced jet. For initial situations propitious to surface frontogenesis, the simulations show a restratification over the whole water column characterized by the amplification in time of the Brunt-Väisälä frequency in the upper oceanic layers. In the absence of surface frontogenesis, such as when the jet is initialized at the middepth of the water column, the restratification is much weaker and slower. Because both simulations have similar kinetic energy and growth rate of baroclinic instability, the results clearly reveal that the restratification is driven by surface frontogenesis in the first case and by vertical PV flux in the interior in the second case. The authors also point out that the dynamics of the interior PV is tightly related to the surface dynamics because of total mass conservation.
\end{abstract}

\section{Introduction}

Several studies have clearly illustrated the role of mesoscale eddies induced by baroclinic instability in setting the oceanic stratification. Marshall et al. (2002) and Karsten et al. (2002) have proposed a theory, based on numerical simulations, to relate the stratification setup over the whole water column in the Antarctic Circumpolar Current to lateral and vertical eddy fluxes. Their studies correspond to the equilibrium reached in the presence of a buoyancy flux and mechanical wind forcing. More specific studies (Samelson and Chapman 1995; Spall 1995; Nurser and Zhang 2000), which focused on the upper $400 \mathrm{~m}$ of the ocean, have documented the shoaling of the mixed layer induced by mesoscale eddies. This is a consequence of frontal instabilities that act on the restratification through the development of baroclinic eddies (see also Cessi and Fantini 2004). Such shoaling of the mixed layer is also illustrated by the increased resolution now available for eddy-resolving general circulation models (e.g., Osch-

Corresponding author address: G. Lapeyre, LMD/IPSL, Ecole Normale Supérieure, 75005 Paris, France.

E-mail: glapeyre@lmd.ens.fr lies 2002). These different results point out that mesoscale eddies and fronts are able to modify the stratification of the ocean through baroclinic instability, in addition to other mechanisms (Vallis 2000; Henning and Vallis 2005).

The study of Hakim et al. (2002) differs from those previously mentioned since it does not invoke baroclinic instability, but only considers the surface dynamics driven by the efficient cascade of density variance that produces smaller and smaller density fronts (since the vertical velocity is zero at the surface). The result is a striking and significant impact of the weak and smallscale density fronts in decreasing the mean density at the ocean surface. The dynamics involved is close to the surface quasigeostrophy (SQG) detailed in Held et al. (1995). SQG is characterized by uniform quasigeostrophic potential vorticity in the interior of the fluid and nonuniform surface density. This kind of balance was revealed by Lapeyre and Klein (2006) to be quite relevant for the mesoscale and submesoscale dynamics of the upper oceanic layers. In particular, their study showed that, for unstable baroclinic flows forced by a surface front, the SQG theory allows one to reconstruct the three-dimensional balanced motions of the upper oceanic layers (i.e., layers in the first $500 \mathrm{~m}$ ) from only 
the knowledge of the surface density field and the large-scale stratification properties. These arguments clearly corroborate the existence in the oceanic upper layers of an efficient cascade of density variance, and therefore of an efficient surface frontogenesis associated with the stirring of the small-scale density fronts. Consequently, one may ask how this surface frontogenesis interacts with the interior dynamics and thereby impacts the time scale of the restratification.

Here we want to present a constraint based on potential vorticity conservation that clearly demonstrates how eddies affect the stratification, at least in the upper oceanic layers. This constraint, in addition to the mass conservation and the omega equation, will unveil how surface frontogenesis affects the restratification processes. Restratification can be viewed as being forced by two different mechanisms: baroclinic instability processes that govern interior PV fluxes and surface frontogenesis (driven by the energetic stirring of density fronts near the ocean surface) that controls strong vertical motions. This will be illustrated with the help of two sets of numerical simulations: one driven by both baroclinic instability and surface frontogenesis (an unstable baroclinic jet with a surface signature) and the other driven by baroclinic instability only (an unstable baroclinic jet with no surface signature).

The paper is organized as follows. First, we discuss the dynamical constraints that apply to the mean density time evolution and to the restratification process by ageostrophic mechanisms. Second, we illustrate these constraints using numerical simulations of an unstable baroclinic jet with different initial conditions. Third, general conclusions for the ocean are drawn.

\section{Dynamical constraints}

\section{a. Potential vorticity conservation constraint}

We first recall the classical equations for a Boussinesq flow on an $f$ plane:

$$
\begin{aligned}
\frac{D \mathbf{u}}{D t}+f_{0} \mathbf{k} \times \mathbf{u} & =-\frac{1}{\rho_{0}} \nabla_{H} P, \\
\frac{D \rho}{D t} & =0, \\
\nabla_{H} \cdot \mathbf{u}+\partial_{z} w & =0, \\
g \rho & =-\partial_{z} P, \text { and } \\
\frac{D}{D t} & =\partial_{t}+\mathbf{u} \cdot \nabla_{H}+w \partial_{z},
\end{aligned}
$$

where $\mathbf{u}$ is the horizontal velocity vector, $P$ is pressure, and $\rho$ is density; $\mathbf{k}$ is the vertical unit vector and $f_{0}$ is the
Coriolis parameter. For this set of equations, the Ertel potential vorticity is

$$
Q=\left(f_{0} \mathbf{k}+\boldsymbol{\nabla} \times \mathbf{u}\right) \cdot \nabla \rho,
$$

where $\boldsymbol{\nabla} \times \mathbf{u}$ is the three-dimensional curl applied to the horizontal velocity $\mathbf{u}$ (i.e., the $3 \mathrm{D}$ vorticity vector associated with the horizontal velocity field). One can also write PV in its flux form:

$$
Q=\nabla_{H} \cdot\left(\rho \mathbf{k} \times \partial_{z} \mathbf{u}\right)+\partial_{z}\left[\rho\left(f_{0}+\zeta\right)\right],
$$

where $\mathbf{k} \times \partial_{z} \mathbf{u}=\left(-\partial_{z} v, \partial_{z} u\right)$ is the horizontal component of the $3 \mathrm{D}$ vorticity vector and $\zeta=\partial_{x} v-\partial_{y} u$ its vertical component (i.e., the vertical relative vorticity). One important property is that Ertel PV is conserved along Lagrangian trajectories in the absence of sources or sinks [see Haynes and McIntyre (1990) for a derivation],

$$
\frac{D Q}{D t}=0
$$

We prefer to rewrite this equation in its flux form,

$$
\partial_{t} Q+\nabla_{H} \cdot(\mathbf{u} Q)+\partial_{z}(w Q)=0,
$$

since the total velocity is nondivergent. Introducing (3) in (4), we obtain

$$
\boldsymbol{\nabla}_{H} \cdot\left[\partial_{t}\left(\rho \mathbf{k} \times \partial_{z} \mathbf{u}\right)+\mathbf{u} Q\right]+\partial_{z}\left\{\partial_{t}\left[\rho\left(f_{0}+\zeta\right)\right]+w Q\right\}=0 .
$$

Now we consider a three-dimensional region of the flow such that $\mathbf{u}$ vanishes at its horizontal boundaries. Applying the horizontal spatial average to (5), the horizontal flux at the meridional boundaries of the domain vanishes. This gives, at any depth,

$$
\partial_{z}\left[\partial_{t}\left\langle\rho\left(f_{0}+\zeta\right)\right\rangle+\langle w Q\rangle\right]=0
$$

where the angle brackets denote the horizontal spatial averaging operator. Integrating this equation in $z$ gives

$$
\partial_{t}\left\langle\rho f_{0}\right\rangle+\partial_{t}\langle\rho \zeta\rangle+\langle w Q\rangle=0 .
$$

This is true, up to a quantity that does not depend on $z$ and that we shall assume to be zero. This happens, for instance, if there is no flow and no density anomaly near the bottom. Equation (7) is the central point of our paper. Muraki et al. (1999) obtained a corollary by integrating the PV conservation equation over the threedimensional space, by which they found that $f_{0}\langle\rho\rangle+$ $\langle\rho \zeta\rangle$ at the surface $(z=0)$ should not depend on time. In contrast, our result, which stems from horizontal averaging, applies at any depth and, as we will show later, has new and profound consequences. Equation (7) states that at any depth the evolution of the horizontally averaged PV integral between this depth and the 
bottom $\left\langle\int^{z} Q d z\right\rangle=\left\langle\rho\left(f_{0}+\zeta\right)\right\rangle$ is balanced only by the vertical PV flux. The major consequence is that the time evolution of mean density depends on two terms:

$$
\partial_{t}\langle\rho\rangle=-\frac{1}{f_{0}}\left(\partial_{t}\langle\rho \zeta\rangle+\langle w Q\rangle\right) .
$$

The first term is the evolution of the correlation between density and vorticity, while the second term is the vertical PV flux. Expanding each variable in a Rossby number expansion, density can be written such that, as $\rho=\rho_{0}+\rho^{0}+\operatorname{Ro} \rho^{1}+\cdots$, where Ro is the Rossby number and $\partial_{z} \rho^{0}=-\rho_{0} N^{2} / g$ is related to the mean stratification (independent of time and $x$ and $y$ ). Also, we have $\zeta=\operatorname{Ro} \zeta^{1}+\cdots$ and $w=\operatorname{Ro}^{2} w^{2}+\cdots$ using the density equation [see Muraki et al. (1999) for a careful derivation]. We find that the mean geostrophic density $\left\langle\rho^{1}\right\rangle$ cannot evolve in time. At the next order, the time evolution of the mean ageostrophic density $\left\langle\rho^{2}\right\rangle$ depends on the geostrophic term $\partial_{t}\left\langle\rho^{1} \zeta^{1}\right\rangle$ and on the PV flux $\left\langle w^{2} q^{1}\right\rangle$ (where $Q=f_{0} \partial_{z} \rho^{0}+\operatorname{Ro} q^{1}$ and $q^{1}=\zeta^{1} \partial_{z} \rho^{0}+$ $\left.f_{0} \partial_{z} \rho^{1}\right)$. Hence it is the geostrophic terms that induce the first-order ageostrophic corrections in the mean density field. This was recognized by Muraki et al. (1999) and Hakim et al. (2002) for the special case of the surface temperature in $\mathrm{QG}^{+1}$ and surface $\mathrm{QG}^{+1}$ theory (where, for the latter, PV is assumed to be uniform in the interior of the fluid). The superscript +1 stands for the next-order correction in the Rossby number included in the $\mathrm{QG}^{+1}$ model as compared with the standard QG model.

Interestingly, the vertical integration of (5) gives another constraint (see appendix A); that is, a particular vector involving the horizontal PV flux must be nondivergent. We will not investigate the implication of this result.

\section{b. Link to frontogenesis}

To better understand the meaning of (8), we propose to examine separately the two terms on its right-hand side. Concerning the term $\partial_{t}\langle\rho \zeta\rangle$, we start from

$$
\frac{D(\rho \zeta)}{D t}=\rho \frac{D \zeta}{D t}=\rho f_{0} \partial_{z} w+\rho \zeta \partial_{z} w+\rho\left(\mathbf{k} \times \partial_{z} \mathbf{u}\right) \cdot \nabla_{H^{w}} .
$$

The last term on the rhs of this equation can be rewritten following the identity

$$
\boldsymbol{\nabla}_{H} \cdot\left(w \mathbf{k} \times \partial_{z} \mathbf{u}\right)=\left(\mathbf{k} \times \partial_{z} \mathbf{u}\right) \cdot \nabla_{H} w-w \partial_{z} \zeta,
$$

which gives

$$
\frac{D(\rho \zeta)}{D t}=\rho f_{0} \partial_{z} w+\rho \boldsymbol{\nabla}_{H} \cdot\left(w \mathbf{k} \times \partial_{z} \mathbf{u}\right)+\rho \partial_{z}(w \zeta) .
$$

Using the definition of Lagrangian derivative

$$
\frac{D(\rho \zeta)}{D t}=\partial_{t}(\rho \zeta)+\nabla_{H} \cdot(\mathbf{u} \rho \zeta)+\partial_{z}(w \rho \zeta)
$$

one has

$$
\begin{aligned}
\partial_{t}(\rho \zeta)= & \nabla_{H} \cdot\left(-\rho \zeta \mathbf{u}+\langle\rho\rangle w \mathbf{k} \times \partial_{z} \mathbf{u}-\langle\rho\rangle f_{0} \mathbf{u}\right)-w \zeta \partial_{z} \rho \\
& +\rho^{\prime} f_{0} \partial_{z} w+\rho^{\prime} \nabla_{H} \cdot\left(w \mathbf{k} \times \partial_{z} \mathbf{u}\right),
\end{aligned}
$$

where $\rho^{\prime}=\rho-\langle\rho\rangle$ is the density anomaly. Now we horizontally average this equation so that the first term vanishes because it is equal to a flux across the borders of the domain and the horizontal velocity vanishes there. Also, we have for the same reason $\langle\rho \zeta\rangle=\left\langle\rho^{\prime} \zeta\right\rangle$, and we see that we have to examine the time evolution of the correlation of the density anomaly with relative vorticity. Finally we obtain

$$
\partial_{t}\left\langle\rho^{\prime} \zeta\right\rangle=-\left\langle w \zeta \partial_{z} \rho\right\rangle+f_{0}\left\langle\rho^{\prime} \partial_{z} w\right\rangle+\left\langle\rho^{\prime} \nabla_{H} \cdot\left(w \mathbf{k} \times \partial_{z} \mathbf{u}\right)\right\rangle .
$$

The first term is the opposite of the vertical flux of the potential vorticity associated with vertical vorticity. The second term is related to the correlation of the density anomaly with the horizontal velocity divergence. The last term should be smaller than the other ones because it arrives at a greater order in a Rossby number expansion.

The term $f_{0}\left\langle\rho^{\prime} \partial_{z} w\right\rangle$ will be the leading term in the rhs of (10) and is actually linked to surface frontogenesis/ frontolysis as recognized by Hakim et al. (2002). Frontogenesis (frontolysis) is the process that leads to formation (destruction) of the horizontal density gradient. Its evolution follows the equation,

$$
\frac{D \boldsymbol{\nabla}_{H} \rho}{D t}=-\left(\boldsymbol{\nabla}_{H} \mathbf{u}\right)^{\mathrm{T}} \boldsymbol{\nabla}_{H} \rho-{ }_{z} \rho \boldsymbol{\nabla}_{H} w,
$$

where $\left(\nabla_{H} \mathbf{u}\right)^{\mathrm{T}}$ is the transposed velocity gradient tensor. The first term on the rhs of (11) is the straining of the density field by the horizontal velocity field, whereas the second term is the flattening (or the steepening) of the isopycnals by the vertical velocity that develops in response. Since $w$ vanishes at the ocean surface, there is an irreversible cascade of surface density variance to small scales, which explains the appearance of small-scale density gradients with large amplitude there. This process is general and is related to the effect of mesoscale eddies that stir, fold, and stretch any tracer that is conserved in a Lagrangian sense (see, e.g., Lapeyre et al. 1999, 2001). However, an increase of the density gradient requires an acceleration of the jet associated with the front in order to keep the thermal wind balance (Hoskins et al. 1978). Near the surface this is done by the horizontal ageostrophic velocity. The divergence of the latter on each side of the jet explains 


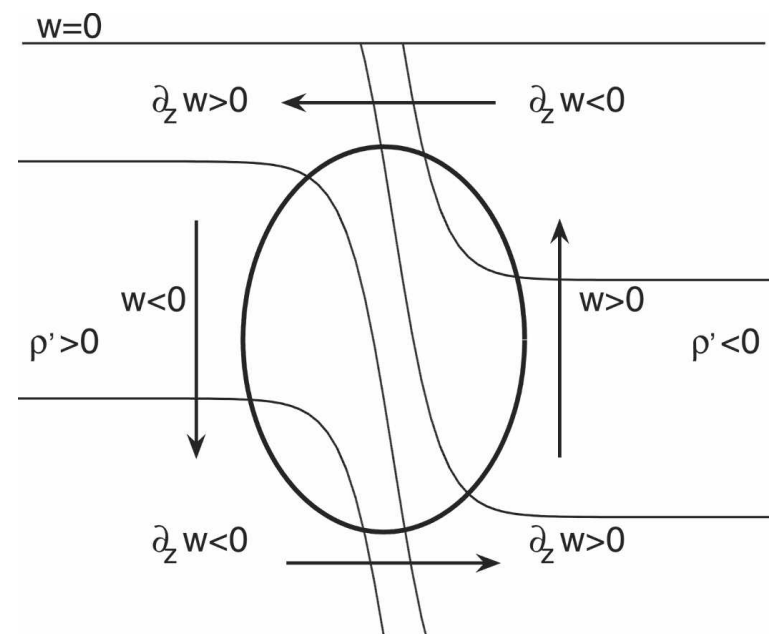

FIG. 1. Sketch of the ageostrophic circulation that develops in response to strengthening of a horizontal density front. The figure corresponds to a vertical cross section through a submesoscale front. Thin lines are isopycnals. The arrows correspond to the ageostrophic circulation. Light fluid is on the right of the figure and dense fluid on the left.

the large values of $\partial_{z} w$ observed near the surface (see Fig. 1), whose effect is to increase the magnitude of relative vorticity (Spall 1995; Nurser and Zhang 2000). The ageostrophic circulation that develops on each side of the density front has a systematic bias that favors the upwelling (downwelling) of light (dense) fluid (see Lévy et al. 2001; Hakim et al. 2002) to release the potential energy associated with the front. As shown in Fig. 1, we expect a positive correlation between $\rho^{\prime}$ and $\partial_{z} w$ at the surface and a negative correlation at depth. This argument is not only valid at the scale of the large- scale front but, more important, at the scale of the submesoscale density gradients that are present in large number in the spatial field. It is primarily these smallscale fronts that will control the efficiency of the restratification.

One may argue that Fig. 1 illustrates the general mechanism of baroclinic instability. However, it is demonstrated below that the value of $\left\langle\rho^{\prime} \partial_{z} w\right\rangle$ crucially depends on the production of small-scale density gradients. First, we recall that the velocity divergence and vertical velocity field are related to frontogenesis through the well-known QG "omega" equation in its Q-vector formulation (Hoskins et al. 1978); that is,

$$
\nabla_{H}^{2} w+\frac{f_{0}^{2}}{N^{2}} \partial_{z}^{2} w=-\frac{2 g}{\rho_{0} N^{2}} \nabla_{H} \cdot \mathbf{Q},
$$

where $w$ is the vertical velocity and $N^{2}$ is the BruntVäisälä frequency,

$$
N^{2}=-\frac{g}{\rho_{0}} \partial_{z}\langle\rho\rangle
$$

(which depends only on $z$ ); the $\mathbf{Q}$ vector is equal to

$$
\mathbf{Q}=\left(\nabla_{H} \mathbf{u}\right)^{\mathrm{T}} \nabla_{H} \rho
$$

Equation (12) states that the vertical velocity field is triggered by the creation of a density gradient by the straining action of oceanic eddies [through the $\mathbf{Q}$ vector that intervenes in (11)]. Its net effect is to oppose to the creation of density gradient in (11) (see Hoskins et al. 1978; Hoskins 1982; Klein et al. 1998).

Using a generalization of the omega equation for the primitive equations similar to Davies-Jones (1991) and Viudez et al. (1996), we can demonstrate that

$$
\begin{aligned}
\left\langle\rho^{\prime} \partial_{z} w\right\rangle= & \int_{-H}^{z}\left(-\frac{2 g}{\rho_{0} f_{0}^{2}}\left\langle\mathbf{Q}^{*} \cdot \nabla_{\mathrm{H}} \rho\right\rangle-\frac{g}{\rho_{0} f_{0}^{2}}\left\langle\partial_{z} \rho \boldsymbol{\nabla}_{H} w \cdot \nabla_{H} \rho\right\rangle+\left\langle\partial_{z} w \partial_{z} \rho\right\rangle-\frac{g}{\rho_{0} f_{0}^{2}}\left\langle\boldsymbol{\nabla}_{H} \rho \cdot \frac{D \mathbf{T I}}{D t}\right\rangle\right. \\
& \left.+\frac{1}{f_{0}}\left\langle\partial_{z} w\left(\mathbf{k} \times \partial_{z} \mathbf{u}\right) \cdot \boldsymbol{\nabla}_{H} \rho\right\rangle\right) d z,
\end{aligned}
$$

where $H$ is the depth of the fluid and $\mathbf{Q}^{*}$ is a modified $\mathbf{Q}$ vector (see appendix B for the derivation). Here thermal imbalance, $\mathbf{T I}$, is the difference between the density gradient vector and the rotated vector of the velocity shear (this difference would be zero if the wind was in thermal wind balance). The first term on the rhs of (13) is the production of density gradients by the deformation field and should be positive. The second term is associated with the flattening of the isopycnals by the vertical velocity field and should be negative. The third term is the effect of stretching on the strati- fication (Nurser and Zhang 2000). Its amplitude may depend on the dynamics of the flow. The last two terms can be assumed small because they intervene at a higher order in a Rossby number expansion. One can expect that the first term on the rhs will dominate since it is the straining action of the eddies on the density field that is at the origin of the ageostrophic circulation. The ageostrophic circulation that develops will counteract its effect through the other terms in (13) so that there will be a modest increase of the density front. If surface frontogenesis dominates, we expect that $\partial_{z} w$ 
will be very large at the ocean surface, so $\left\langle\rho^{\prime} \partial_{z} w\right\rangle$ will be the dominant term to balance the density gradient production. This has the direct consequence that a correlation will be created between the velocity divergence and the surface density field if small-scale fronts are able to develop at the ocean surface. The term $\partial_{t}\left\langle\rho^{\prime} \zeta\right\rangle / f_{0}$ will be positive at the surface, implying a decrease of $\langle\rho\rangle$ there and an increase of $N^{2}$. Stirring of density structures and resulting surface frontogenesis is thus seen to promote the restratification, and the sketch of Fig. 1 applies at submesoscales where the density gradients have a larger increase in magnitude than at the scale of the jet.

\section{c. Interior dynamics}

The other term that modifies the mean density in (8) is the vertical PV flux. This term does not intervene in the restratification when considering surface $\mathrm{QG}^{+1} \mathrm{dy}$ namics (Hakim et al. 2002). For this reason we relate it to the interior dynamics. The vertical PV flux can be decomposed in different parts:

$$
\begin{aligned}
\langle w Q\rangle= & \langle w\rangle f_{0} \partial_{z}\langle\rho\rangle+f_{0}\left\langle w \partial_{z} \rho^{\prime}\right\rangle+\langle w \zeta\rangle \partial_{z}\langle\rho\rangle+\left\langle w \zeta \partial_{z} \rho^{\prime}\right\rangle \\
& +\left\langle w\left(\mathbf{k} \times \partial_{z} \mathbf{u}\right) \cdot \nabla_{H} \rho\right\rangle .
\end{aligned}
$$

The first term vanishes because the vertical velocity is zero, on average (since it comes from the horizontal divergence of $\mathbf{u}$ and $\mathbf{u}$ is assumed to vanish at the domain boundaries). We thus have

$$
\begin{aligned}
\langle w Q\rangle= & f_{0}\left\langle w \partial_{z} \rho^{\prime}\right\rangle+\langle w \zeta\rangle \partial_{z}\langle\rho\rangle+\left\langle w \zeta \partial_{z} \rho^{\prime}\right\rangle \\
& +\left\langle w\left(\mathbf{k} \times \partial_{z} \mathbf{u}\right) \cdot \nabla_{H} \rho\right\rangle .
\end{aligned}
$$

The first term represents the vertical flux of vortex stretching. The second and third terms represent the vertical flux of relative vorticity weighted by the stratification. They also appear in the equation for $\partial_{t}\left\langle\rho^{\prime} \zeta\right\rangle$ $[(10)]$ but with the opposite sign. This suggests that they do not contribute directly to the restratification process but that they intervene in the interaction between frontogenesis and the interior dynamics. The main difference with (10) is that the term contributing to the restratification is no longer $f_{0}\left\langle\rho^{\prime} \partial_{z} w\right\rangle$, but is $f_{0}\left\langle w \partial_{z} \rho^{\prime}\right\rangle$.

We can evaluate the sign of $\langle w \zeta\rangle_{z}\langle\rho\rangle$ from the sketch shown in Fig. 1 . Assuming the balance, $D \zeta / D t \approx f_{0} \partial_{z} w$, the velocity divergence will be associated with relative vorticity anomalies such that $\zeta / f_{0}$ will be of the same sign as $\partial_{z} w$. Owing to $\partial_{z}\langle\rho\rangle<0$ because of the stratification, this implies that $\langle w \zeta\rangle \partial_{z}\langle\rho\rangle / f_{0}$ will be positive at the surface and negative at the bottom. Since the mean potential vorticity is $f_{0} \partial_{z}\langle\rho\rangle$, this is associated with downward PV flux in the upper layers and upward flux in the lower layers.
Because of (8) and the mass conservation, it can be shown that the restratification terms (10) and (14) are not independent from each other. Indeed, from the conservation of the total mass of the fluid, one must have

$$
\partial_{t} \int_{-H}^{0}\langle\rho\rangle d z=0 .
$$

When integrating (8) over depth, this implies that

$$
\int_{-H}^{0} \partial_{t}\left\langle\rho^{\prime} \zeta\right\rangle d z+\int_{-H}^{0}\langle w Q\rangle d z=0 .
$$

The discussion in the last section showed that the first term is forced by frontogenesis to be negative (positive) in the Southern (Northern) Hemisphere. Therefore, the second term must compensate in order to satisfy this constraint. As $w_{\text {rms }}$ vanishes at the surface and reaches its maximum at middepth (near the crossing of the first baroclinic mode), $\langle w Q\rangle$ will be maximum in the interior of the fluid and of opposite sign to $\partial_{t}\left\langle\rho^{\prime} \zeta\right\rangle$. Since the sign of $f_{0}\left\langle w \partial_{z} \rho^{\prime}\right\rangle$ cannot be evaluated using arguments based on Fig. 1 , we infer that it is $f_{0}\left\langle w \partial_{z} \rho^{\prime}\right\rangle$ that will be modified to satisfy the balance because its sign is not determined by frontogenesis processes. This indicates that the process of interaction between the surface and the interior of the fluid is made through the vortex stretching term.

\section{Numerical simulations}

\section{a. Description}

We have shown that mesoscale eddies and submesoscale fronts may affect the restratification by two mechanisms: the first one is related to surface frontogenesis (associated with stirring of small-scale density structures in the upper layers) and the second one to baroclinic instability in the interior through vertical PV fluxes. To better evaluate the importance of these processes, we use primitive equation simulations of the nonlinear development of a baroclinic unstable jet. The setting considered in this work is close to the one considered by others (e.g., Samelson and Chapman 1995; Nurser and Zhang 2000; Rivière et al. 2004) of an initially laminar balanced jet that freely evolves through nonlinear baroclinic destabilization. This setup allows one to characterize the eddy adjustment of an initial state built by large-scale forcing (Gutowski et al. 1989). Our initial state is characterized by a vertically uniform Brunt-Väisälä profile to the north and south of the jet. We also choose a simulation in the Southern Hemisphere on the $\beta$ plane with $f_{0}=-1.17 \times 10^{-4} \mathrm{~s}^{-1}<0$ and parameters appropriate for the Antarctic Circum- 
(a) Simulation $\mathrm{A}$

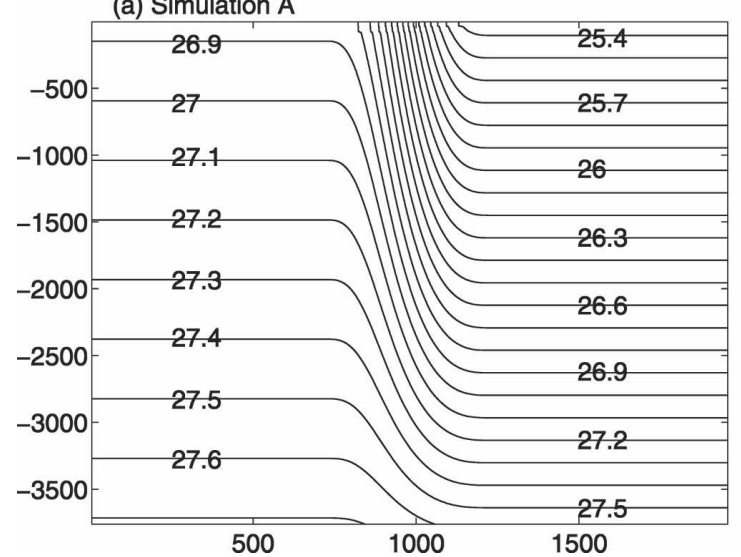

(b) Simulation B

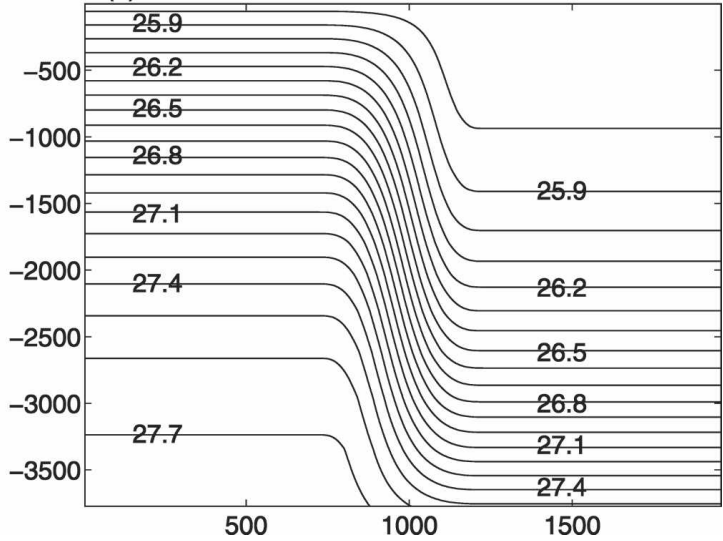

FIG. 2. Vertical section of the zonally averaged density $\left(\mathrm{kg} \mathrm{m}^{-3}\right)$ for (a) experiment $\mathrm{A}$ and (b) experiment B; abscissa is in kilometers, and ordinate is in meters.

polar Current. The setup is similar to Rivière et al. (2004) and Lapeyre and Klein (2006) except for its basic stratification, which serves to highlight the role of ageostrophic eddies. At last, we free ourselves of any diabatic effect by focusing on nonlinear eddy effects in the absence of any surface fluxes. The model is a $z$ coordinate model with free-slip boundary conditions at the northern and southern walls. The domain is a square, $2000 \mathrm{~km} \times 2000 \mathrm{~km}$, with a depth of $4000 \mathrm{~m}$.

Two sets of simulations are considered. The first one (experiment A) consists of a jet with an initial meridional density gradient maximum at the surface, and the second one (experiment B) consists of a jet with a meridional density gradient that is zero at the surface and with a maximum at $2000 \mathrm{~m}$ (cf. the meridional vertical cross sections of the two initial density fields in Figs. 2a,b). The Eady instability growth rate $f_{0} \partial_{z} u / N$ and the horizontal density gradient have a similar amplitude for the two simulations at the initial time (see Fig. 7a). This leads to a growth in time of the kinetic energy that is similar between the two sets of simulations (see Fig. 3). The baroclinic instability that develops is of a different kind in each simulation: for the first set of simulations, the initial meridional PV gradient does not change sign in the interior but is opposite to the surface vertical shear so that the criterion of instability is met (Bretherton 1966). For the second set, the initial meridional PV gradient changes sign in the interior of the fluid, satisfying the classical criterion of baroclinic instability.

For each set of simulations, we varied the numerical resolution. The horizontal resolution will be either 3.8 $\mathrm{km} \times 3.8 \mathrm{~km}(\mathrm{~A} 3.8$ or B3.8) or $6 \mathrm{~km} \times 6 \mathrm{~km}(\mathrm{~A} 6$ or B6). We will examine mainly the results of $\mathrm{A} 6$ and $\mathrm{B} 6$. There are 35 levels with a vertical grid spacing ranging from $4 \mathrm{~m}$ near the surface to $400 \mathrm{~m}$ near the bottom. Using different horizontal resolutions allows one to grasp the sensitivity of the restratification to frontogenesis since the resolution impacts the magnitude of the density fronts.

To have some guidance on the dynamics present in the different simulations, we can examine the vorticity field at the surface for the two sets of runs (see Figs. $4 a, c)$. After 50 days of simulation, it displays classical patterns associated with the evolution of a large-scale jet. Eddies of 150-km diameter have developed on each side of the jet and have stirred filaments with high relative vorticities. At this time the simulations possess the same qualitative features except for, maybe, less intense surface structures in experiment $\mathrm{B}$, but we will come back to this point later. Each simulation was carried out over 100 days, at which time the eddies approach the northern and southern boundaries of the domain. As the lateral boundary condition is different from our assumptions, we will consider diagnostics af-

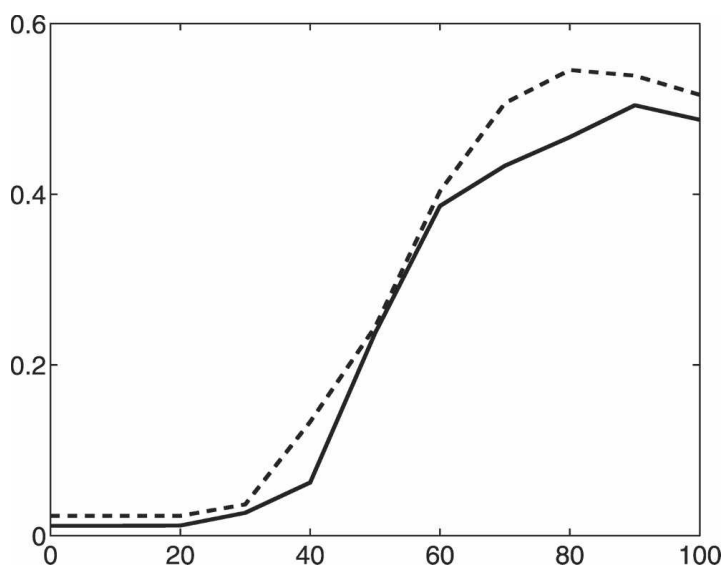

FIG. 3. Evolution of the total kinetic energy $\left(\mathrm{m}^{2} \mathrm{~s}^{-2}\right)$ averaged over the whole fluid for simulation A6 (dashed curve) and simulation B6 (continuous curve) as a function of time (days). 

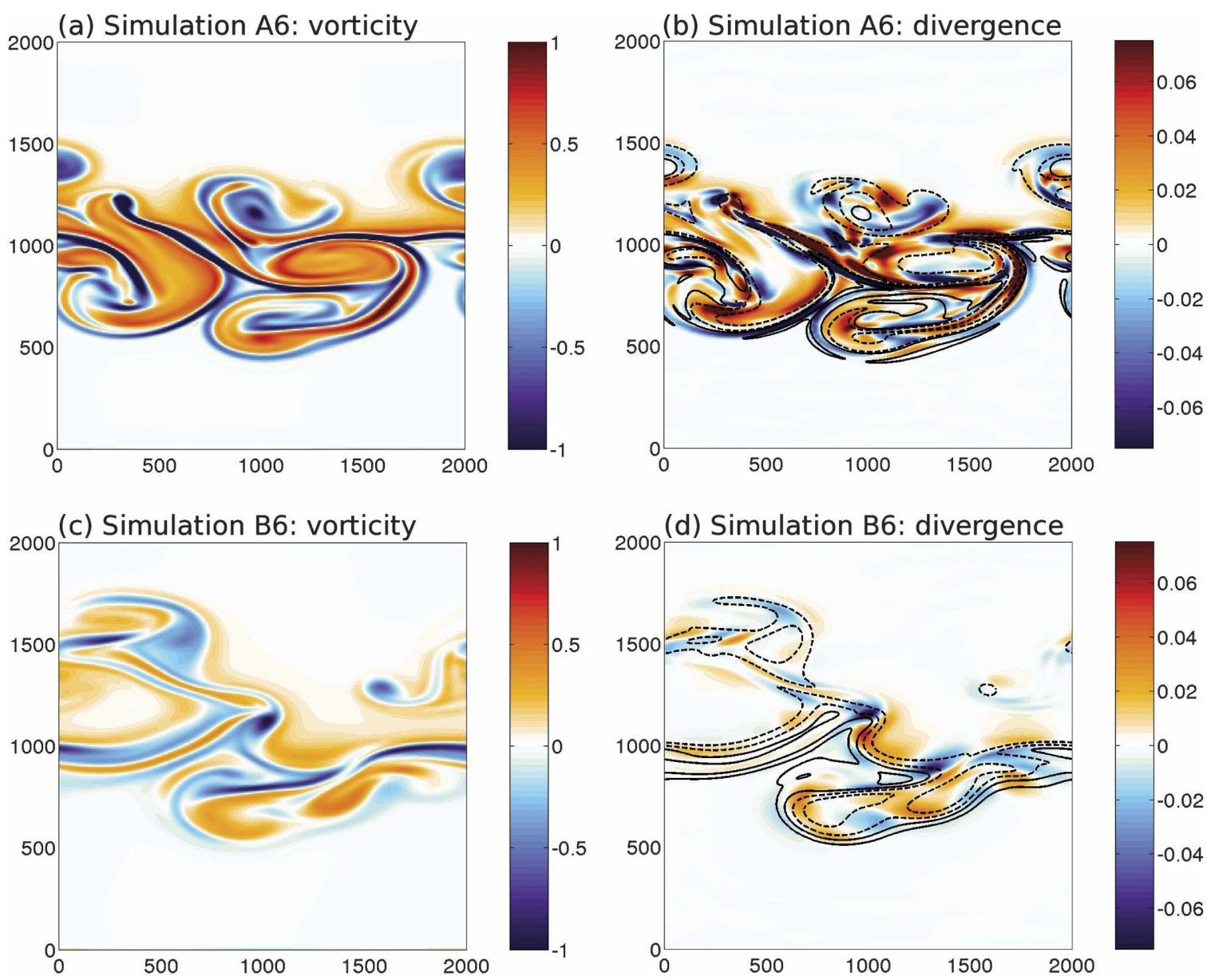

FIG. 4. (a), (c) Relative vorticity (divided by $\left|f_{0}\right|$ ) at the surface for simulation A6 and B6, and (b), (d) horizontal velocity divergence (divided by $\left|f_{0}\right|$ ) at ocean surface. Superimposed are contours of the density anomaly field (continuous curves for positive $\rho^{\prime}$ and dashed for negative $\rho^{\prime}$ ) at the surface for A6 and at $500 \mathrm{~m}$ for B6.

ter 50 days of integration, that is, well before the eddies have approached the boundaries of the domain. We have checked that similar results can be obtained at an earlier or a later time.

\section{b. Restratification analysis}

After 50 days, the eddies have begun to modify the mean density (Figs. 5a and 5b). Lighter fluid has been lifted and denser fluid has downwelled so that, on average, the upper layers have less density than initially and the lower layers have greater density. The isopycnals tend to flatten to release potential energy, which is accompanied with an upwelling of light fluid and a downwelling of heavy fluid. Through this process, the available potential energy is transformed not only into eddy kinetic energy but also into mean potential energy. In the two sets of simulations, potential energy was released, but not at the same vertical level (deeper in experiments B than in A). One notable difference between the two simulations is that the density modification is much stronger for A6 that for B6 and it occurs mainly at the surface in A6.

The stratification parameter, given by the rms of the Brunt-Väisälä frequency divided by $\left|f_{0}\right|$, is shown in Fig. 6 for the two sets of simulations. In experiment A6, there is an increase in stratification above $2000 \mathrm{~m}$ and a decrease below (Fig. 6a). The restratification is maximum at the surface and decreases with depth, resulting in the emergence of an exponential profile in the Brunt-Väisälä frequency $N^{2}$ that amplifies with time. For experiment B6, the restratification is quite different: there is actually a destratification of the upper layers (above $800 \mathrm{~m}$ ) and a restratification in the interior (Fig. 6b). One striking result is the correspondence between the vertical profile of $N^{2}$ and the profile of the density gradient magnitude (cf. Figs. 7a and 6a,b). In experiment $\mathrm{A} 6$, the $\mathrm{rms}$ of $\left|\nabla_{H} \rho^{\prime}\right|$ increases quite strongly at the surface (by a factor 5.36) while $N^{2}$ also 

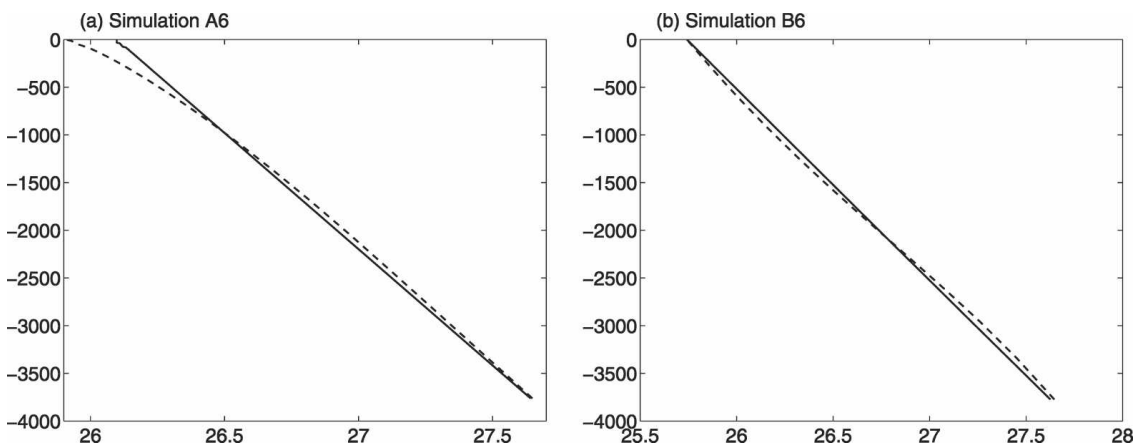

FIG. 5. Vertical profile of the horizontal average of density anomaly $\left(\mathrm{kg} \mathrm{m}^{-3}\right)$, initially (continuous curve) and after 50 days of integration (dashed curve) for (a) simulation A6 and (b) simulation B6.

increases by a factor 2.38 . In experiment $\mathrm{B} 6$, the density gradients increase in rms in the interior by a factor 1.92 (at $2000 \mathrm{~m}$ ) while $N^{2}$ increases by a factor 1.13 . Furthermore, the intensified density gradients at the surface in A6 are associated with an increase of both the rms of the relative vorticity (Fig. 7b) and that of the velocity divergence (Fig. 7c). The significant increase of these two quantities in the upper layers is the signature of the frontogenesis mechanisms triggered by the stirring of the density gradients near the surface. We clearly see that this signature has a direct impact on the restratification process.

To elucidate the mechanisms of the restratification induced by the eddies, Fig. 8 a represents the different terms involved in (7) for experiment A6. First, $f_{0} \partial_{t}\langle\rho\rangle$ is positive (remember $f_{0}<0$ ) in the upper layers and negative below $1000 \mathrm{~m}$. In the first $500 \mathrm{~m}$, it is essentially balanced by $\partial_{t}\left\langle\rho^{\prime} \zeta\right\rangle$. This is another indication of the importance of frontogenesis processes in this simulation. Below $1500 \mathrm{~m}, f_{0} \partial_{t}\langle\rho\rangle$ is balanced by the vertical PV flux. The amplitude of the restratification is quite strong as the mean value of $\partial_{t}\langle\rho\rangle$ over 50 days is 0.002 $\mathrm{kg} \mathrm{m}^{-3} \mathrm{day}^{-1}$, averaging over the first $700 \mathrm{~m}$. This is equivalent to a mean surface heat flux of $C_{p} \int \partial_{t}\langle\rho\rangle d z / \alpha$ $\approx 300 \mathrm{~W} \mathrm{~m}^{-2}$ acting during 50 days over a depth of 700 $\mathrm{m}$ (with $\alpha$ the thermal expansion coefficient). This value, which is larger than the usual mean surface heat fluxes, shows that a substantial density forcing is imposed by baroclinic eddies in the upper layers.

For experiment B6, the mean density evolution in this simulation is mostly driven by the PV vertical flux (Fig. 8b). This flux has the same order of magnitude as the PV vertical flux in A6, which means that it mainly depends on interior processes that are similar between the two experiments (remember that the mean Eady growth rate or the mean kinetic energy are almost the same). The smallness of $\partial_{t}\left\langle\rho^{\prime} \zeta\right\rangle$ can be explained by the absence of a significant production of density gradients in the upper layers and therefore of surface frontogenesis as discussed in the next section. Indeed, $\partial_{t}\left\langle\rho^{\prime} \zeta\right\rangle$ reaches only $0.0022 \mathrm{~kg} \mathrm{~m}^{-3}$ day $^{-2}$ at its maximum value around $1000 \mathrm{~m}$, a value quite modest in comparison with the other simulation $\left(0.012 \mathrm{~kg} \mathrm{~m}^{-3}\right.$ day $^{-2}$ at the surface).

\section{c. Frontogenesis analysis}

The difference between simulations A and B is the presence or absence of density fronts at the surface.
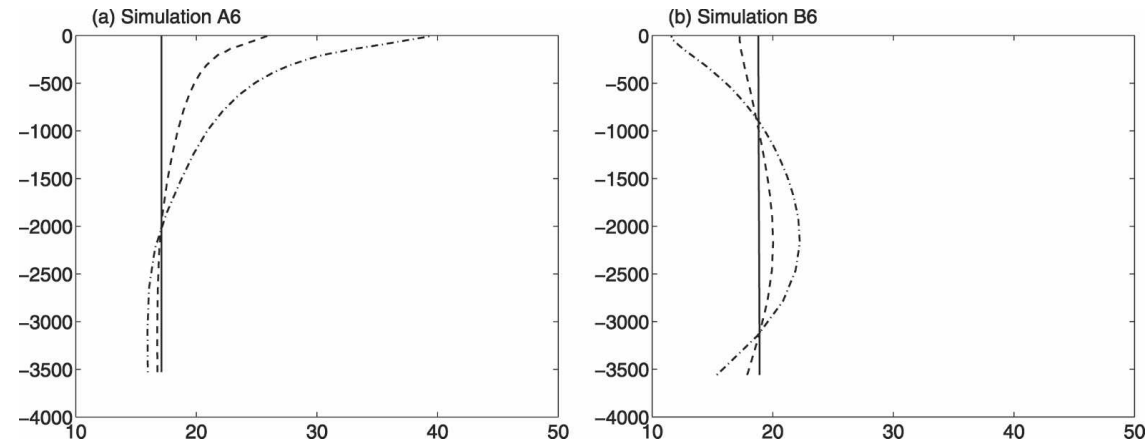

FIG. 6. Mean profiles of $N /\left|f_{0}\right|$, initially (continuous curve), after 50 days (dashed curve), and after 100 days (dash-dotted curve) for (a) simulation A6 and (b) simulation B6. 

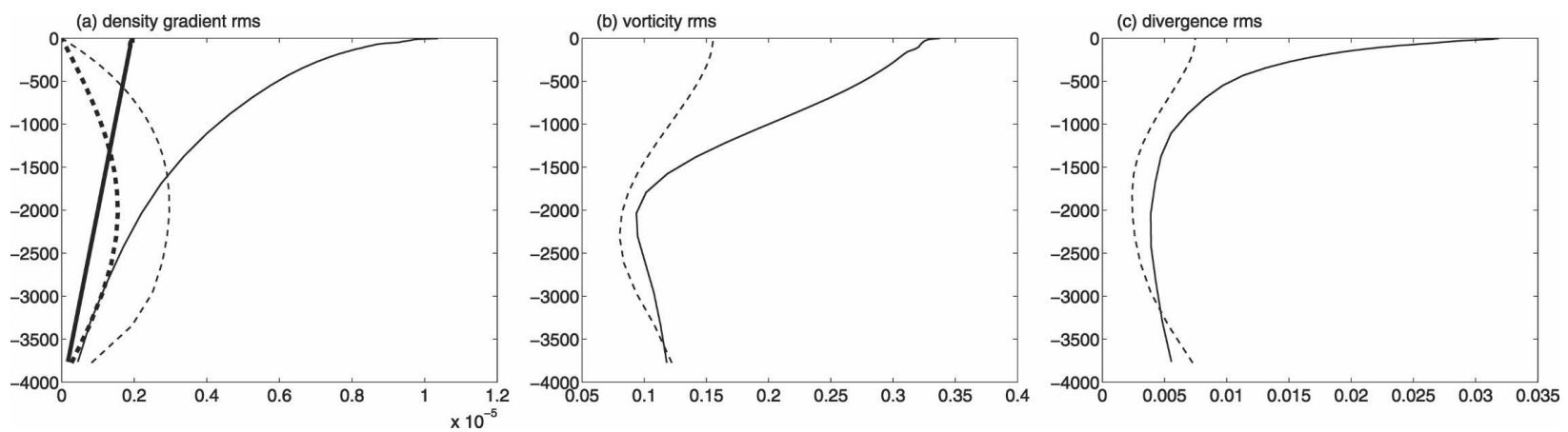

FIG. 7. (a) Vertical profile of the rms of the density gradient magnitude $\left(\mathrm{kg} \mathrm{m}^{-4}\right)$ for simulation A6 (continuous curve) and B6 (dashed curve), initially (thick curves) and after 50 days (thin curves). (b) Vertical profile of rms relative vorticity divided by $\left|f_{0}\right|$ after 50 days. (c) Vertical profile of rms horizontal velocity divergence divided by $\left|f_{0}\right|$ after 50 days. Curves in (b) and (c) have the same meaning as in (a).

Actually, the rms of the density gradient $\left|\nabla_{H} \rho\right|$ vanishes at the surface in B6 and grows at depth, but much less rapidly than in simulation A6 (see Fig. 7a). Using the arguments developed in section $2 b$, we expect that it is the frontogenesis that is responsible for the stronger restratification in A than in B. The absence of surface frontogenesis also explains the smaller mean Rossby number $\left(\zeta_{\mathrm{rms}}\left|f_{0}\right|=0.15\right.$ at the surface) in B6 relative to the one in A6 $\left(\zeta_{\mathrm{rms}}\left|f_{0}\right|=0.32\right)$. This is also confirmed by the maximum Rossby numbers, which are, respectively, $|\zeta|_{\max } /\left|f_{0}\right|=1.04$ in B6 and $|\zeta|_{\max }\left|f_{0}\right|=2.98$ in A6. The Rossby number increases by a factor of 2 between the two simulations, but the time derivative of the mean density increases by a factor of 5 , which highlights the dramatic effect of the surface frontogenesis. On the other hand, the eddy kinetic energy and the Burger number $\left[\mathrm{Bu}=N^{2} H^{2} / f_{0}^{2} L^{2}=\left|f_{0}\right| \mathrm{Ro}^{2} /\left(\nabla_{H} \cdot \mathbf{u}\right)_{\mathrm{rms}} \approx 3.5\right]$ are similar for the two simulations (to define the Burger number we used the density equation and the thermal wind balance).
Figure 9 helps to evaluate how surface frontogenesis is involved in the restratification process. The figure shows the three different terms on the rhs of (10). For simulation A6, the dominant term in the first $500 \mathrm{~m}$ is $f_{0}\left\langle\rho^{\prime} \partial_{z} w\right\rangle$, as expected. The correlation between the density anomaly and velocity divergence, as well as the magnitude of both quantities (Figs. 7a and 7c), is thus central to the decrease of the mean surface density. As can be seen in Figs. 4b and 4d, one can frequently observe at the scale of filaments negative (positive) density anomalies associated with positive (negative) anomalies in velocity divergence (examine the filaments near $x=y=500 \mathrm{~km}$ in Fig. 4b). This correlation was also noted by Hakim et al. (2002) in simulations of $\mathrm{SQG}^{+1}$ turbulent flows with uniform QG PV. However, their results only apply to the surface density field using a system of balanced equations, whereas we observe this correlation for a few hundred meters in a full primitive equation simulation. Concerning simulation B6, we see that all terms in (10) are an order of mag-
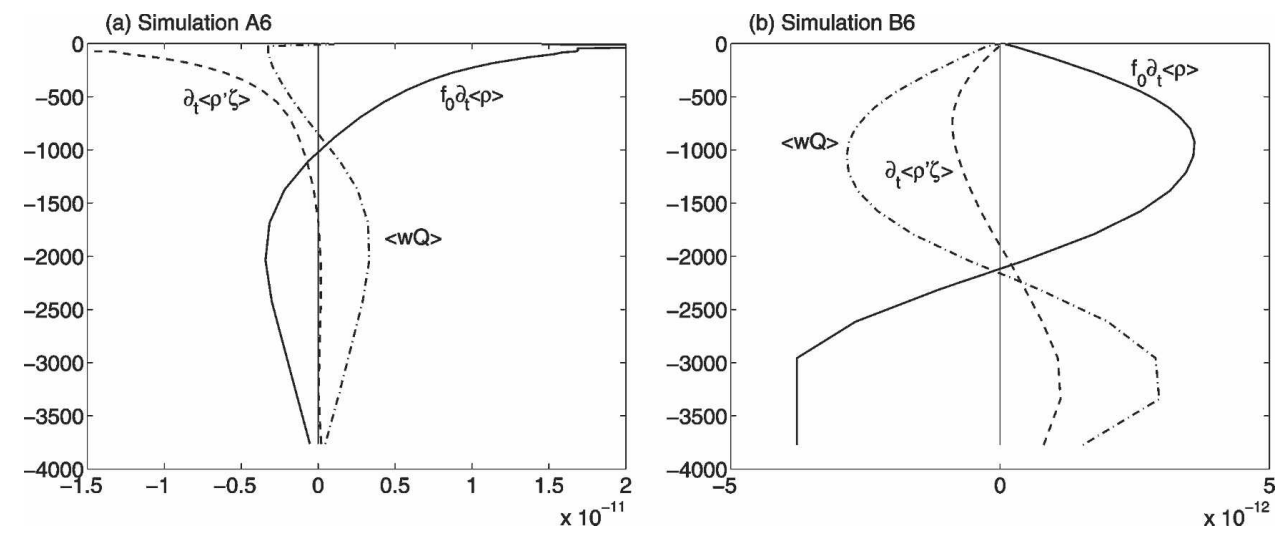

FIG. 8. Terms $\left(\mathrm{kg} \mathrm{m}^{-3} \mathrm{~s}^{-2}\right)$ constituting (7) as a function of depth for (a) simulation A6 and (b) simulation B6. 
(a) Simulation $\mathrm{A} 6$

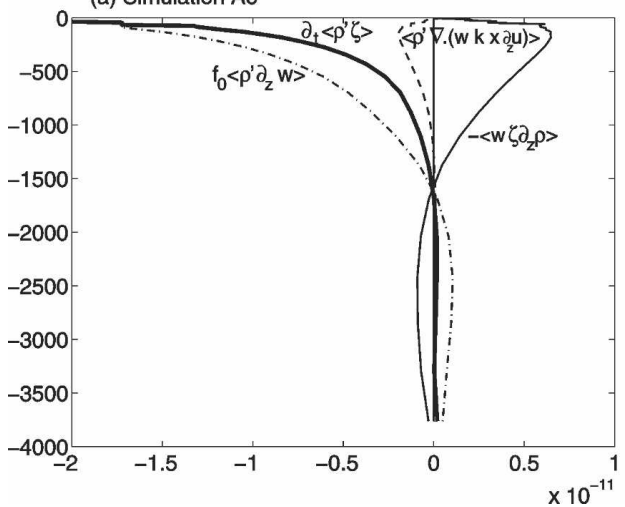

(b) Simulation B6

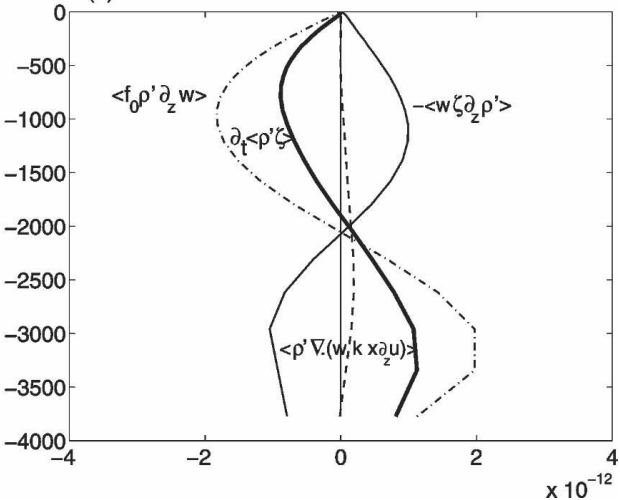

FIG. 9. As in Fig. 8 but constituting (10).

nitude smaller than in A6. Therefore, the presence of surface frontogenesis in A6 substantially increases the magnitude of these terms. One reason is that a very strong ageostrophic circulation (associated with large horizontal divergence $\boldsymbol{\nabla}_{H} \cdot \mathbf{u}$ ) has to develop to reestablish the thermal wind balance in simulation A6. This circulation is, in some sense, inefficient to prevent the growth of the amplitude of the surface fronts since $w$ vanishes at the surface. This results in large amplitudes for the density gradient magnitude and ageostrophic circulation. On the contrary, in simulation B6, the vertical velocity is able to counteract the growth of the interior fronts, which explains the smaller magnitude of the fronts there (see Figs. 7a and 7c).

To further highlight the relation between surface frontogenesis and the term $\left\langle\rho^{\prime} \partial_{z} w^{\prime}\right\rangle$, we can first examine the different terms in (13) that represent the constraint between vertical velocities and frontogenesis. Figure 10a shows that, as expected, it is the production of the density gradient by the deformation field (RHS 1) that dominates. All other terms tend to oppose it near the surface for the two simulations. For experiment A6, the term $\left\langle\rho^{\prime} \partial_{z} w^{\prime}\right\rangle$ is second in amplitude, and this demonstrates that surface frontogenesis will constrain restratification quite strongly when surface fronts are present. It is interesting to note that in experiment B (Fig. 10b), it is the term $\int_{-H}^{z}\left\langle\partial_{z} \rho^{\prime} \partial_{z} w^{\prime}\right\rangle d z$ that is the second to dominate. This term is equivalent to $\left\langle\rho^{\prime} \partial_{z} w^{\prime}\right\rangle$, but takes into account the interior dynamics (through the term $\partial_{z} \rho^{\prime}$ related to vortex stretching). The production of fronts is not efficient in modifying the restratification when there is no surface frontogenesis.

When changing the numerical resolution from $6 \mathrm{~km}$ (in experiment A6) to $3.8 \mathrm{~km}$ (in experiment A3.6), we observed that the surface enstrophy increases by $36 \%$ and the square of the surface density gradient $\left\langle\left|\nabla_{H} \rho\right|^{2}\right\rangle$ increases by $53 \%$. This has some impact on the restratification since the term $-\left\langle\rho^{\prime} \partial_{z} w\right\rangle$ increases by $21 \%$ at the surface. As a result, $N / \mid f_{0}$ l passes from 34.2 to 38.3 at the surface at 70 days. We clearly see that increasing the resolution leads to increasing the magnitude of the density fronts near the surface and of the restratification
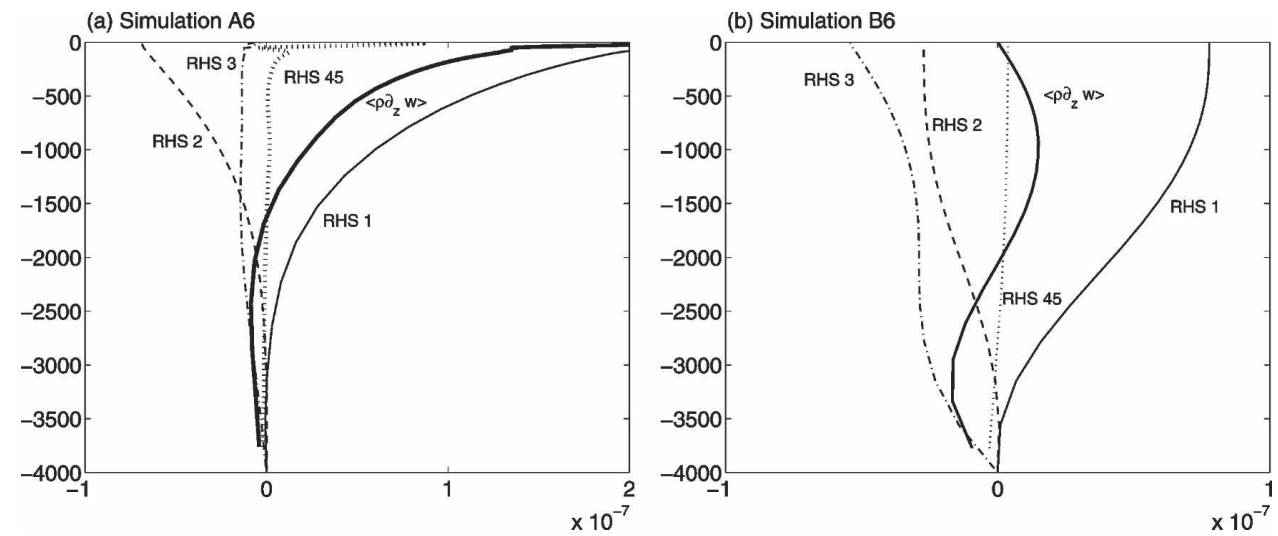

FIG. 10. As in Fig. 8 but constituting (13); the thick curve is $\left\langle\rho^{\prime} \partial_{z} w\right\rangle$. Terms named RHS stand for each term on the rhs of (13). 

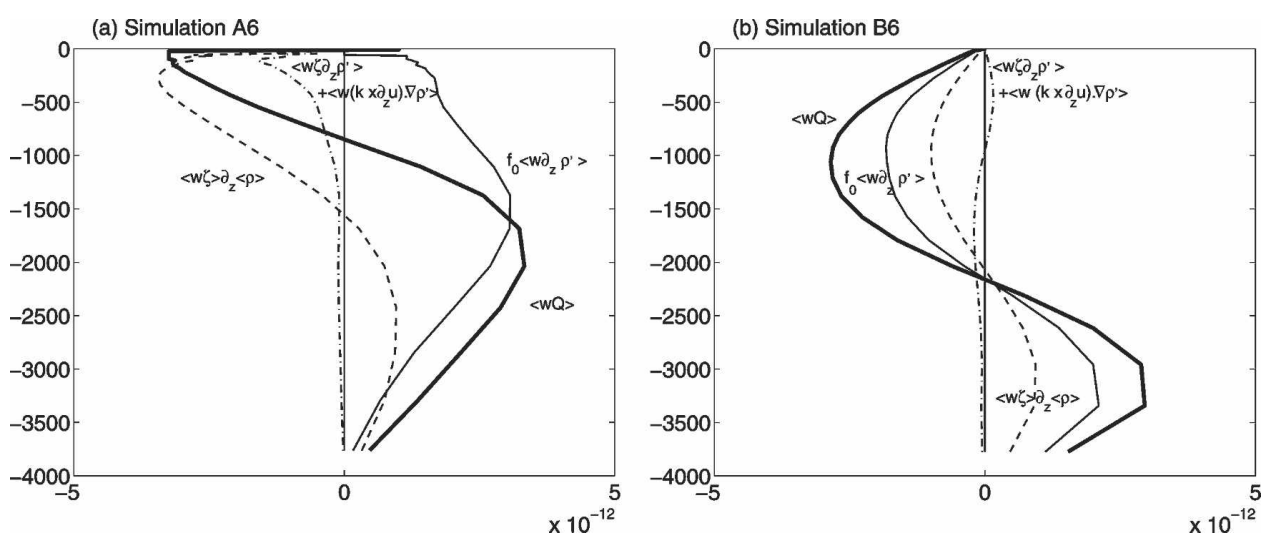

FIG. 11. Decomposition of $\langle w Q\rangle\left(\mathrm{kg} \mathrm{m}^{-3} \mathrm{~s}^{-2}\right)$ following (14) as a function of depth for (a) simulation A6 and (b) simulation B6.

there. On the contrary, in the experiment without surface frontogenesis, the value of $N /\left|f_{0}\right|$ is similar between experiments B6 and B3.8.

The different comparisons between simulations A and $\mathrm{B}$ have clearly revealed that the intensified production of density gradients in the upper layers and the resulting surface frontogenesis play a strong role in the restratification of the oceanic upper layers. When one allows the production of density gradients by the straining action of eddies, the oceanic layers restratify much more rapidly (time scale of months) that when only interior geostrophic motions are present (time scale of years).

\section{d. Interior analysis}

The different terms in (14) that contribute to the modification of the mean density by the PV flux are shown in Figs. 11a,b for the two experiments. For experiment $\mathrm{A} 6$, the dominant terms are the vertical fluxes of vortex stretching [second term on the rhs of (14)] and relative vorticity (third term). Near the surface, it is the relative vorticity flux that dominates, whereas below $1000 \mathrm{~m}$, in the "interior," it is the vortex stretching flux that dominates. We see that the vortex stretching term $f_{0}\left\langle w \partial_{z} \rho^{\prime}\right\rangle$ does not change sign with depth in this experiment. This has to do with mass conservation as we have shown that the vertical PV flux must compensate the density evolution owing to surface frontogenesis through relation (15). Because $\partial_{t}\left\langle\rho^{\prime} \zeta\right\rangle$ is mostly negative in $\mathrm{A} 6,\langle w Q\rangle$ must be positive when integrated vertically, and this is done through the term $f_{0}\left\langle w \partial_{z} \rho^{\prime}\right\rangle$.

When surface frontogenesis is weak, such as in experiment $\mathrm{B} 6$, the term that dominates in (14) is $-f_{0}\left\langle w \partial_{z} \rho^{\prime}\right\rangle$. Contrary to A6, it changes sign with depth, being negative in the first $2000 \mathrm{~m}$ and positive below. The term $\langle w \zeta\rangle \partial_{z}\langle\rho\rangle$ is smaller here because relative vorticity is much weaker than in the other experiment (see Fig. 7b), due to the absence of surface frontogenesis. The sign of this term is in agreement with our prediction using the simple argument based on Fig. 1 since it is negative in the upper layers and positive below. Also, relation (15) transforms into a constraint of zero total PV flux, as can be observed in Fig. 11b. It thus seems that interior dynamics is free to evolve in this experiment, with a weak interaction with surface dynamics.

\section{Conclusions}

We have shown that the conservation of potential vorticity strongly constrains the time evolution of the mean density at a given depth. This result has been obtained using the primitive equations and in the absence of sources and sinks of PV. It was tested using initial value simulations in free decay. Eddies and small-scale fronts affect the restratification through two effects: first, surface frontogenesis, induced by the efficient stirring of density fronts near the surface, increases stratification in the upper layers by creating a correlation between density and relative vorticity; second, vertical PV flux can increase or decrease the stratification in the interior of the fluid. The numerical simulations reveal that small-scale surface fronts have a major impact on the restratification process. Moreover, the mass conservation adds another constraint on the system that couples the surface and the interior dynamics: the interior PV fluxes have to adjust to balance the continuous frontogenesis at the surface that lifts up light fluid. The constraint on the time evolution of the mean density is modified in the presence of a source and sink in the PV equation. However, as shown in the appendix $\mathrm{C}$, they do not contribute directly to the re- 
stratification (except maybe for the mean density forcing), and our result will hold when the flow is subject to forcing.

The modification of the stratification observed here can be related to the baroclinic adjustment theory as first described by Gutowski (1985); he suggested that the flow tends to decrease its meridional gradient of potential vorticity by adjusting the stratification $\left(N^{2}\right)$ in order to suppress baroclinic instability. Indeed, Gutowski et al. (1989) (see also Barry et al. 2000) observed an increase in surface stratification with an initial jet configuration close to ours (i.e., with a density gradient concentrated near the surface), but in an atmospheric context. Here our analytical and numerical results show that it is not only the adjustment by the PV fluxes (associated with the baroclinic instability) but also the surface frontogenesis that control the restratification. More important, our results about these surface frontogenesis effects do not refer to any instability of the density fronts in the upper layers [as invoked in Samelson and Chapman (1995) and Nurser and Zhang (2000)], but only to the properties of submesoscale ageostrophic circulations that develop to counteract the stirring and, therefore growth, of horizontal density gradients.

How should the frontogenesis mechanism act in the real ocean? In regions such as the Antarctic Circumpolar Current (ACC), the flow is characterized by the presence of a strong surface density front [see, e.g., Marshall et al. (1993) and references therein]. The strong nonlinear interactions associated with this front strongly suggest that frontogenesis will play an important role there. We performed a simulation with a setting closed to the ACC [with a vertical profile of $N^{2}$ similar to Rivière et al. (2004)] and found that the mechanism linked to frontogenesis dominates, such as in simulation A. We observed also a density increase of $1.3 \times 10^{-3} \mathrm{~kg} \mathrm{~m}^{-3}$ day $^{-1}$ at the ocean surface corresponding to a typical mean surface heat flux of $11 \mathrm{~W}$ $\mathrm{m}^{-2}$. The density increase is observed only in the first $250 \mathrm{~m}$. This heat flux is smaller than the fluxes due to external heating or cooling by the atmosphere but is not negligible.

We think that the mechanisms highlighted here may be applicable also to the problem of the sharpening of the tropopause (Haynes et al. 2001) because an exponential form of the Brunt-Väisälä frequency is often observed and because frontogenesis is invoked in the dynamics (Wirth 2004).

Acknowledgments. The authors thank Pascal Rivière for helpful comments about this work. All simulations were performed on the supercomputers of CNRS at
IDRIS. This work is supported by IFREMER and the Centre National de la Recherche Scientifique.

\section{APPENDIX A}

\section{Dynamical Constraints}

Based on the PV conservation, another dynamical constraint can be obtained. Integrating (5) vertically from top to bottom gives

$$
\begin{aligned}
& \int_{-H}^{0} \boldsymbol{\nabla}_{H} \cdot\left[\partial_{t}\left(\rho \mathbf{k} \times \partial_{z} \mathbf{u}\right)+\mathbf{u} Q\right] d z+\left.\partial_{t}\left[\rho\left(f_{0}+\zeta\right)\right]\right|_{z=0} \\
& \quad-\left.\partial_{t}\left[\rho\left(f_{0}+\zeta\right)\right]\right|_{z=-H}=0,
\end{aligned}
$$

because vertical velocity vanishes at top and bottom of the domain. Next, we have

$$
\partial_{t}\left(f_{0} \rho\right)=-\boldsymbol{\nabla}_{H} \cdot\left(f_{0} \rho \mathbf{u}\right)-f_{0} \partial_{z}(w \rho) .
$$

Using (9), we obtain

$$
\partial_{t}\left[\left(f_{0}+\zeta\right) \rho\right]=\nabla_{H} \cdot\left(-\rho \zeta \mathbf{u}-\rho f_{0} \mathbf{u}\right)
$$

at $z=0$ and $z=-H$. If there is no motion at the ocean bottom, we obtain

$$
\boldsymbol{\nabla}_{H} \cdot\left\{\int_{-H}^{0}\left[\partial_{t}\left(\rho \mathbf{k} \times \partial_{z} \mathbf{u}\right)+\mathbf{u} Q\right] d z-\left.\rho\left(f_{0}+\zeta\right) \mathbf{u}\right|_{z=0}\right\}=0 .
$$

This implies that the quantity

$$
\int_{-H}^{0}\left[\partial_{t}\left(\rho \mathbf{k} \times \partial_{z} \mathbf{u}\right)+\mathbf{u} Q\right] d z-\left.\rho\left(f_{0}+\zeta\right) \mathbf{u}\right|_{z=0}
$$

is nondivergent. The vertical shear integrated over depth is thus seen to be controlled by the PV flux and its surface analog.

\section{APPENDIX B}

\section{Modified Q Vector Derivation}

The principle to obtain (13) can first be explained for its QG counterpart. The idea is to multiply the QG omega equation [(12)] by $\rho^{\prime}$, and to integrate by parts horizontally or vertically. Then, one obtains a QG version of (13). For the primitive equations, we will start by deriving a quantity similar to the thermal wind imbalance since this equation allows one to derive the omega equation (Hoskins et al. 1978; Giordani and Planton 2000). The thermal wind balance would imply that

$$
\boldsymbol{\nabla}_{H} \rho=\frac{\rho_{0} f_{0}}{g} \mathbf{k} \times \partial_{z} \mathbf{u}
$$


We note TI (thermal imbalance) as the difference of the two terms, $\nabla_{\mathrm{H}} \rho-\left(\rho_{0} f_{0} / g\right) \mathbf{k} \times \partial_{z} \mathbf{u}$. Because the vertical shear obeys the equation

$$
\frac{D \partial_{z} \mathbf{u}}{D t}=-\left(\partial_{z} \mathbf{u} \cdot \nabla_{H}\right) \mathbf{u}-\partial_{z} w \partial_{z} \mathbf{u}-f_{0} \mathbf{k} \times \partial_{z} \mathbf{u}+\frac{g}{\rho_{0}} \nabla_{H} \rho
$$

and the density gradient obeys (11), it follows that

$$
\begin{aligned}
\frac{D \mathbf{T I}}{D t}= & -\left(\boldsymbol{\nabla}_{H} \mathbf{u}\right)^{\mathrm{T}}\left(\boldsymbol{\nabla}_{H} \rho+\frac{f_{0} \rho_{0}}{g} \mathbf{k} \times \partial_{z} \mathbf{u}\right)-\partial_{z} \rho \boldsymbol{\nabla}_{H^{w}} \\
& +\frac{\rho_{0} f_{0}}{g} \partial_{z} w \mathbf{k} \times \partial_{z} \mathbf{u}-\frac{\rho_{0} f_{0}^{2}}{g} \partial_{z} \mathbf{u}-f_{0} \mathbf{k} \times \nabla_{H} \rho .
\end{aligned}
$$

As explained by Hoskins et al. (1978) and Klein et al. (1998) in the QG theory, the first term on the rhs acts to destroy the thermal wind balance. It is balanced by the second and third term, which will tend to reestablish this balance so that $D \mathbf{T I} / D t$ will remain small. Multiplying this equation by $\nabla_{H} \rho$, the last term vanishes. The term $\left(\rho_{0} f_{0}^{2} / g\right) \partial_{z} \mathbf{u} \cdot \nabla_{H} \rho$ can be rewritten using the identity

$$
\begin{aligned}
\boldsymbol{\nabla}_{H} \cdot\left(\rho \partial_{z} \mathbf{u}\right) & =\partial_{z} \mathbf{u} \cdot \nabla_{H} \rho+\rho \partial_{z} \nabla_{H} \cdot \mathbf{u} \\
& =\partial_{z} \mathbf{u} \cdot \nabla_{H} \rho-\rho \partial_{z}^{2} w \\
& =\partial_{z} \mathbf{u} \cdot \nabla_{H} \rho-\partial_{z}\left(\rho \partial_{z} w\right)+\partial_{z} \rho \partial_{z} w .
\end{aligned}
$$

One has

$$
\begin{aligned}
\boldsymbol{\nabla}_{H} \rho \cdot \frac{D \mathbf{T I}}{D t}= & -2 \boldsymbol{Q}^{*} \cdot \boldsymbol{\nabla}_{H} \rho-\partial_{z} \rho \boldsymbol{\nabla}_{H} w \cdot \nabla_{H} \rho+\frac{\rho_{0} f_{0}^{2}}{g} \partial_{z} w \partial_{z} \rho \\
& -\frac{\rho_{0} f_{0}^{2}}{g} \partial_{z}\left(\rho \partial_{z} w\right)+\frac{\rho_{0} f_{0}}{g} \partial_{z} w\left(\mathbf{k} \times \partial_{z} \mathbf{u}\right) \cdot \nabla_{H} \rho \\
& -\frac{\rho_{0} f_{0}^{2}}{g} \nabla_{H} \cdot\left(\rho \partial_{z} \mathbf{u}\right),
\end{aligned}
$$

where $\mathbf{Q}^{*}$ equals

$$
\mathbf{Q}^{*}=\frac{1}{2}\left(\boldsymbol{\nabla}_{H} \mathbf{u}\right)^{*}\left(\boldsymbol{\nabla}_{H} \rho+\frac{f_{0} \rho_{0}}{g} \mathbf{k} \times \partial_{z} \mathbf{u}\right) ;
$$

Q* is a generalized $\mathbf{Q}$ vector that takes into account the effect of the deformation field on the density gradient and the thermal wind. Now, taking a horizontal average, the last term on the rhs of (B1) vanishes such that

$$
\begin{aligned}
\left\langle\boldsymbol{\nabla}_{H} \rho \cdot \frac{D \mathbf{T I}}{D t}\right\rangle= & -2\left\langle\mathbf{Q}^{*} \cdot \nabla_{H} \rho\right\rangle-\left\langle\partial_{z} \rho \boldsymbol{\nabla}_{H} w \cdot \nabla_{H} \rho\right\rangle \\
& +\frac{\rho_{0} f_{0}^{2}}{g}\left\langle\partial_{z} w \partial_{z} \rho\right\rangle-\frac{\rho_{0} f_{0}^{2}}{g} \partial_{z}\left\langle\rho \partial_{z} w\right\rangle \\
& +\frac{\rho_{0} f_{0}}{g}\left\langle\partial_{z} w\left(\mathbf{k} \times \partial_{z} \mathbf{u}\right) \cdot \nabla_{H} \rho\right\rangle .
\end{aligned}
$$

Integrating from the bottom to a depth $z$ and assuming that at the bottom $\rho^{\prime}=0$, we finally obtain

$$
\begin{aligned}
-\frac{g}{\rho_{0} f_{0}^{2}} \int_{-H}^{z}\left(2\left\langle\mathbf{Q}^{*} \cdot \nabla_{H} \rho\right\rangle+\left\langle\boldsymbol{\nabla}_{H} \rho \cdot \frac{D \mathbf{T I}}{D t}\right\rangle\right) d z= & \left\langle\rho^{\prime} \partial_{z} w\right\rangle-\int_{-H}^{z}\left\langle\partial_{z} w \partial_{z} \rho\right\rangle d z+\frac{g}{\rho_{0} f_{0}^{2}} \int_{-H}^{z}\left\langle\partial_{z} \rho \boldsymbol{\nabla}_{H^{w}} \cdot \boldsymbol{\nabla}_{H} \rho\right\rangle d z \\
& -\frac{1}{f_{0}} \int_{-H}^{z}\left\langle\partial_{z} w\left(\mathbf{k} \times \partial_{z} \mathbf{u}\right) \cdot \nabla_{H} \rho\right\rangle d z .
\end{aligned}
$$

Such an equation shows that frontogenesis theory exerts a constraint between the production of the density gradient and the ageostrophic circulation that develops to maintain the thermal wind balance. In particular, the vertical velocity field will tend to organize in relation to the density field so that the terms on the lhs will balance the first term on the rhs and that the thermal imbalance will remain small.

\section{APPENDIX C}

\section{Modified Potential Vorticity Equation Including Friction}

If $\mathbf{F}$ is the frictional force applied to the momentum equations and $B$ is an external heat source (or sink), such that the equations of motions are

$$
\begin{aligned}
\frac{D \boldsymbol{u}}{D t}+f_{0} \mathbf{k} \times \mathbf{u} & =-\frac{1}{\rho_{0}} \nabla_{H} P+\mathbf{F} \text { and } \\
\frac{D \rho}{D t} & =B,
\end{aligned}
$$

the potential vorticity equation is modified such that

$$
\begin{aligned}
\partial_{t} Q+\nabla_{H} \cdot(\mathbf{u} Q)+\partial_{z}(w Q)= & \nabla_{H} \cdot\left(\rho \mathbf{k} \times \partial_{z} \mathbf{F}+B \mathbf{k}\right. \\
& \left.\times \partial_{z} \mathbf{u}\right)+\partial_{z}\left[\rho \mathbf{k} \cdot\left(\nabla_{H} \times \mathbf{F}\right)\right. \\
& +B(\zeta+f)] .
\end{aligned}
$$

The constraint (7) becomes

$$
\partial_{t}\left\langle\rho f_{0}\right\rangle+\partial_{t}\langle\rho \zeta\rangle+\langle w Q\rangle=\left\langle\rho\left(\partial_{x} F_{y}-\partial_{y} F_{x}\right)+B\left(\zeta+f_{0}\right)\right\rangle
$$

if frictional forces vanish at the boundaries of the domain. The new terms are the correlation of the vorticity forcing with the density anomaly and the correlation of 
the vertical vorticity with the density forcing. To identify the effect of the sources or sinks in the restratification, we need also to know how $\partial_{t}\left\langle\rho^{\prime} \zeta\right\rangle$ is modified by these sources or sinks. One would also find a term in the rhs of (10), equal to

$$
\left\langle\rho\left(\partial_{x} F_{y}-\partial_{y} F_{x}\right)+B \zeta\right\rangle .
$$

Therefore, the evolution of $\partial_{t}\left\langle\rho f_{0}\right\rangle$ will only be modified by $\left\langle B f_{0}\right\rangle$ and not by the other terms. This could be expected since the mean density budget can be written as

$$
\partial_{t}\langle\rho\rangle+\partial_{z}\langle w \rho\rangle=\langle B\rangle
$$

from the density equation in its flux form. Therefore, the sources or sinks will not have a direct effect if $\langle B\rangle=$ 0 . They can still have an indirect effect by modifying the dynamics and limiting, for instance, the ageostrophy of the flow. We therefore conclude that the processes we describe in free decay will remain valid in a forced simulation in statistical equilibrium. In such a situation, even if $\partial_{t}\langle\rho\rangle$ will remain close to zero, the frontogenesis terms of the PV terms will still lead to restratification.

\section{REFERENCES}

Barry, L., G. C. Craig, and J. Thuburn, 2000: A GCM investigation into the nature of baroclinic adjustment. J. Atmos. Sci., 57, 1141-1155.

Bretherton, F. P., 1966: Critical layer instability in baroclinic flows. Quart. J. Roy. Meteor. Soc., 92, 325-334.

Cessi, P., and M. Fantini, 2004: The eddy-driven thermocline. $J$. Phys. Oceanogr., 34, 2642-2658.

Davies-Jones, R., 1991: The frontogenetical forcing of secondary circulations. Part I: The duality and generalization of the $\mathbf{Q}$ vector. J. Atmos. Sci., 48, 497-509.

Giordani, H., and S. Planton, 2000: Modeling and analysis of ageostrophic circulation over the Azores oceanic front during the SEMAPHORE experiment. Mon. Wea. Rev., 128, 2270-2287.

Gutowski, W. J., 1985: Baroclinic adjustment and midlatitude temperature profiles. J. Atmos. Sci., 42, 1733-1745.

— L. E. Branscome, and D. A. Stewart, 1989: Mean flow adjustment during life cycles of baroclinic waves. J. Atmos. Sci., 46, 1724-1737.

Hakim, G. J., C. Snyder, and D. J. Muraki, 2002: A new surface model for cyclone-anticyclone asymmetry. J. Atmos. Sci., 59, 2405-2420.

Haynes, P. H., and M. E. McIntyre, 1990: On the conservation and impermeability theorems for potential vorticity. J. Atmos. Sci., 47, 2021-2031.

_ J. F. Scinocca, and M. D. Greenslade, 2001: Formation and maintenance of the extratropical tropopause by baroclinic eddies. Geophys. Res. Lett., 28, 4179-4182.

Held, I. M., R. T. Pierrehumbert, S. T. Garner, and K. L. Swanson, 1995: Surface quasi-geostrophic dynamics. J. Fluid Mech., 282, 1-20.
Henning, C. C., and G. K. Vallis, 2005: The effects of mesoscale eddies on the stratification and transport of an ocean with a circumpolar channel. J. Phys. Oceanogr., 35, 880-896.

Hoskins, B. J., 1982: The mathematical theory of frontogenesis. Annu. Rev. Fluid Mech., 14, 131-151.

—, I. Draghici, and H. C. Davies, 1978: A new look at the $\omega$-equation. Quart. J. Roy. Meteor. Soc., 104, 31-38.

Karsten, R., H. Jones, and J. Marshall, 2002: The role of eddy transfer in setting the stratification and transport of a circumpolar current. J. Phys. Oceanogr., 32, 39-54.

Klein, P., A.-M. Tréguier, and B. L. Hua, 1998: Threedimensional stirring of thermohaline fronts. J. Mar. Res., 56, 589-612.

Lapeyre, G., and P. Klein, 2006: Dynamics of the upper oceanic layers in terms of surface quasigeostrophy theory. J. Phys. Oceanog., 36, 165-176.

- —, and B. L. Hua, 1999: Does the tracer gradient vector align with the strain eigenvectors in 2-D turbulence? Phys. Fluids, 11A, 3729-3737.

— B. B. Hua, and P. Klein, 2001: Dynamics of orientation of gradients of active and passive scalars in two-dimensional turbulence. Phys. Fluids, 13A, 251-264.

Lévy, M., P. Klein, and A.-M. Tréguier, 2001: Impact of submesoscale physics on production and subduction of phytoplankton in an oligotrophic regime. J. Mar. Res., 59, 535565.

Marshall, J., D. Olbers, H. Ross, and D. Wolf-Gladrow, 1993: Potential vorticity constraints on the dynamics and hydrography of the Southern Ocean. J. Phys. Oceanogr., 23, 465487.

, H. Jones, R. Karsten, and R. Wardle, 2002: Can eddies set ocean stratification? J. Phys. Oceanogr., 32, 26-38.

Muraki, D. J., C. Snyder, and R. Rotunno, 1999: The next-order corrections to quasi-geostrophic theory. J. Atmos. Sci., 56, $1547-1560$.

Nurser, A. J. G., and J. W. Zhang, 2000: Eddy-induced mixed layer shallowing and mixed layer/thermocline exchange. $J$. Geophys. Res., 105, $21851-21868$.

Oschlies, A., 2002: Improved representation of upper-ocean dynamics and mixed layer depths in a model of the North Atlantic on switching from eddy-permitting to eddy-resolving grid resolution. J. Phys. Oceanogr., 32, 2277-2298.

Rivière, P., A.-M. Tréguier, and P. Klein, 2004: Effects of bottom friction on nonlinear equilibration of an oceanic baroclinic jet. J. Phys. Oceanogr., 34, 416-432.

Samelson, R. M., and D. C. Chapman, 1995: Evolution of the instability of a mixed-layer front. J. Geophys. Res., 100, 67436759.

Spall, M. A., 1995: Frontogenesis, subduction, and cross-front exchange at upper ocean fronts. J. Geophys. Res., 100, 25432557.

Vallis, G. K., 2000: Large-scale circulation and production of stratification: Effects of wind, geometry, and diffusion. $J$. Phys. Oceanogr., 30, 933-954.

Viudez, A., J. Tintore, and R. L. Haney, 1996: About the nature of the generalized omega equation. J. Atmos. Sci., 53, 788795.

Wirth, V., 2004: A dynamical mechanism for tropopause sharpening. Meteor. Z., 13, 477-484. 